\title{
Multi-epoch time-resolved photometry of the eclipsing polar
} CSS081231:071126+440405

\author{
A.D. Schwope ${ }^{1, \star}$, F. Mackebrandt ${ }^{1}$, B.D. Thinius ${ }^{2}$, C. Littlefield ${ }^{3,4}$, P. Garnavich ${ }^{3}$, A. Oksanen ${ }^{5}$, and \\ T. Granzer ${ }^{1}$ \\ 1 Leibniz-Institut für Astrophysik Potsdam (AIP), An der Sternwarte 16, 14482 Potsdam, Germany \\ 2 Inastars Observatory, Hermann-Struve-Str. 10, 14469 Potsdam, Germany \\ 3 Department of Physics, University of Notre Dame, 225 Nieuwland Science Hall, Notre Dame, IN 46556, USA \\ 4 Department of Astronomy, Wesleyan University, Middletown, CT 06459, USA \\ ${ }^{5}$ Hankasalmi Observatory, Jyväskylän Sirius ry, Vertaalantie 419, 40270 Palokka, Finland
}

Received December 4, 2014, accepted January 21, 2015

Key words stars: individual: CSS081231:071126+440405 - binaries: eclipsing - stars: cataclysmic variables

\begin{abstract}
The eclipsing polar CSS081231:071126+440405 turned bright $\left(V_{\max } \sim 14.5\right)$ in late 2008 and was subsequently observed intensively with small and medium-sized telescopes. A homogeneous analysis of this comprehensive dataset comprising 109 eclipse epochs is presented and a linear ephemeris covering the five years of observations, about 24000 orbital cycles, is derived. Formally this sets rather tight constraints on the mass of a hypothetical circumbinary planet, $M_{\mathrm{pl}} \leq 2 \mathrm{M}_{\text {Jup }}$. This preliminary result needs consolidation by long-term monitoring of the source. The eclipse lasts $433.08 \pm 0.65 \mathrm{~s}$, and the orbital inclination is found to be $i=79.3^{\circ}-83.7^{\circ}$. The centre of the bright phase displays accretion-rate dependent azimuthal shifts. No accretion geometry is found that explains all observational constraints, suggesting a complex accretion geometry with possible pole switches and a likely non-dipolar field geometry.
\end{abstract}

(c) 2014 WILEY-VCH Verlag GmbH \& Co. KGaA, Weinheim

\section{Introduction}

Jupiter-sized planets on Jupiter-like wide orbits were discovered in recent years around eclipsing close binary stars with white-dwarf primaries and main-sequence secondary stars (WDMS binaries). Such discoveries were possible via the light-travel time effect, i.e. via periodic shifts of the eclipses of the central binary, and they require precise eclipse timing and long monitoring campaigns. Suitable target stars are relatively rare, to date less than 100 such objects are known. Circumbinary planets were found orbiting accreting and non-accreting binaries of WDMS type, i.e. cataclysmic binaries (CVs) and pre-CVs (see e.g. Beuermann et al. 2010; Qian et al. 2011).

Orbital period variations in those objects may come from different sources, they may be caused by activity cycles of the secondary stars or by circumbinary planets. In addition, magnetic braking of the binary and gravitational radiation will occur but their strength is not enough to make an observable impact on the time-scale of a scientific career. Some objects show strong, quasi-regular variability in their eclipse arrival times that cannot be explained by current models (see the claimed discovery of a planetary system around HU Aqr; Bours et al. 2014; Goździewski et al. 2012; Horner et al. 2011; Oian et al. 2011; Schwarz et al. 2009; Schwope \& Thinius 2014; Wittenmyer et al. 2012).

^ Corresponding author: e-mail: aschwope@ aip.de
CSS081231:071126+440405 (henceforth J071126) was discovered as variable transient-like object in the Catalina Real-Time Transient Survey (Drake et al. 2009) when it suddenly turned bright from $V \sim 18$ to $V \sim 14.5$ in the fall of the year 2008. Following vsnet-alert \#10867 Denisenko \& Korotkiy (2008, reported in CVnet discussion \#1208) obtained the first time-resolved photometric observations. They confirmed a maximum brightness of 14.4 and reported the occurrence of deep eclipses, which were used to determine an orbital period of $116.6 \mathrm{~m}$. The shape of the light-curve led them to classify the object as an eclipsing polar. In its high state it was found to be similarly bright as the template eclipsing polar HU Aquarii.

Polars are magnetic cataclysmic variable stars. Contrary to normal CVs the strong magnetic moment of the accreting white dwarf keeps both stars in synchronous rotation. The magnetic field suppresses the formation of an accretion disk. Instead the accreted matter is guided towards the magnetic pole(s) where the accretion luminosity eventually is released, part of it as strongly polarized cyclotron radiation. Polars are sources of soft and hard X-rays that are originating from regions close to the magnetic poles. The absence of an accretion disk results in short response times to changes of the mass transfer rate that may occur on time scales from seconds to years. During low states, when the accretion stream is reduced to a trickle, the optical light is dominated by the photospheres of the stellar constituents plus perhaps some cyclotron radiation from residual accretion. During high accretion states the spectral energy dis- 
Table 1 Facilities used in this study and their observational set-up

\begin{tabular}{|c|c|c|c|c|c|c|c|}
\hline Observatory & $\begin{array}{r}\text { Aperture } \\
(\mathrm{cm})\end{array}$ & Detector & Filter & $\begin{array}{r}T_{\exp } \\
\text { (s) }\end{array}$ & \# frames & $\begin{array}{r}\text { latency } \\
(\mathrm{s})\end{array}$ & $\begin{array}{r}\text { syst. unc. } \\
\text { (s) }\end{array}$ \\
\hline $\mathrm{HO}$ & 40 & SBIG STL-1001E & clear & 30 & 6730 & 0.0 & 3.00 \\
\hline Nonndorf & 25.4 & Videocam. WAT-120N & - & 2 & 10218 & 0.0 & 2.56 \\
\hline VATT 2009 & 180 & VATT4K & $\mathrm{B} / \mathrm{V}$ & $20,30 / 30$ & $426,321 / 608$ & 0.0 & 0.0 \\
\hline VATT 2010 & 180 & VATT4K & $\mathrm{U} / \mathrm{B}$ & $30 / 30$ & $526 / 517$ & 0.0 & 0.0 \\
\hline VATT 2010 & 180 & VATT4K & $\mathrm{V}$ & $10,15,30$ & $112,549,206$ & 0.0 & 0.0 \\
\hline VATT 2013 & 180 & VATT4K & R/vilS* & $10 / 20$ & $306 / 222$ & 0.0 & 0.0 \\
\hline STELLA 2012 & 120 & CCD & g & 15 & 543 & 3.0 & 0.1 \\
\hline STELLA 2013 & 120 & $\mathrm{CCD}$ & $\mathrm{g}$ & 60 & 724 & 3.0 & 0.1 \\
\hline IOP & 36 & SBIG ST-8 3 & Astrometrik CLS & 3 & 6845 & 0.0 & 0.0 \\
\hline SCT28 & 28 & SBIG ST-8 3 & clear & $8,10,15,30$ & $6304,1353,64,438$ & 1.5 & 0.0 \\
\hline SLKT & 80 & SBIG STL-1001 3 & Luminance & 2 & 222 & 2.6 & 0.0 \\
\hline
\end{tabular}

* the Vilnius $\mathrm{S}$ filter has maximum transparency at $\mathrm{H} \alpha$ and a FWHM of $180 \AA$.

tribution of the binary is dominated by accretion-induced radiation which outshines the photospheric radiation.

Bright eclipsing objects like HU Aqr and now J071126 are prime targets for detailed investigations addressing the accretion physics and are qualified for high-time resolution observations to determine precise eclipse times.

The first printed publications on J071126 are by Thorne et al. (2010) and Katysheva \& Shugarov (2012) who were refining the eclipse ephemeris and discussing the shape of the light-curve in low, intermediate and high states of accretion. The object occasionally shows a pronounced pre-eclipse dip due to the intervening accretion stream or accretion curtain, whose location in phase in principle helps to uncover the accretion geometry.

At its maximum brightness the variable star is within the reach of small telescopes and was targeted by amateurs at several sites. Amateur equipment may allow short integration times and their results can be used to study the LTTeffect. Lower signal-to-noise per image compared to professional telescopes can be compensated by 'infinite' observation time available at private observatories.

Motivated by recent reports of circumbinary planets, by the large brightness of J071126, and by the availability of a large body of archival data made available via the internet, we decided to obtain further time-resolved photometric observations of J071126 and analyze the archival and new own data in a homogeneous way to derive a reliable ephemeris for the centre of the eclipse and to search for possible systematic deviations that could give a hint for the occurrence of an unseen companion.

In Sect. 2 we describe both new and archival observations and how the data were treated to determine eclipse times for the individual epochs. In Sect. 3.1 we derive an updated eclipse ephemeris, in Sect. 3.2 we analyse properties of the optical light curves and derive binary parameters and discuss the possible accretion geometry in Sect.4.

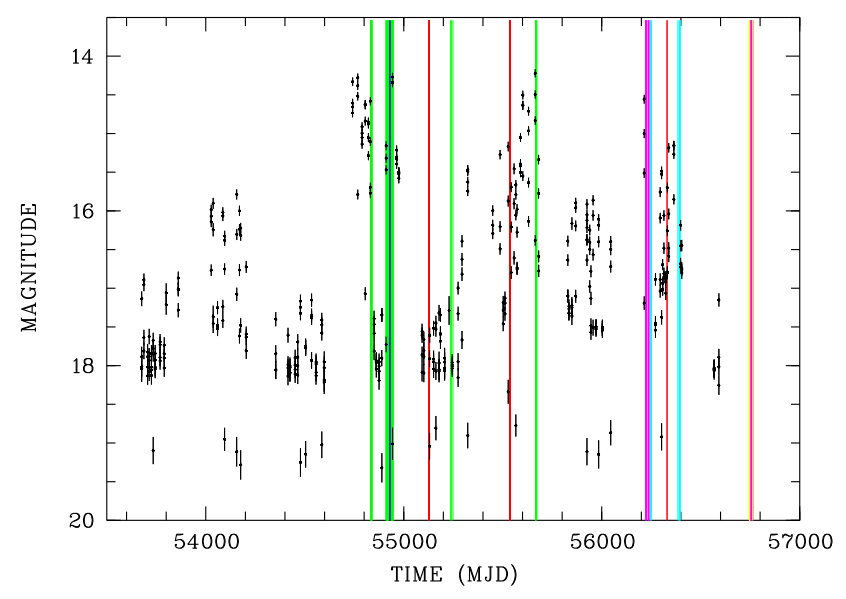

Fig. 1 Long-term light curve of CSS081231:071126+440405 derived from the CSSdatabase. Vertical lines indicate the epochs used here for the determination of the binary period. Color indicates the equipment used (green - HO, blue - Dangl, red VATT, cyan - STELLA, magenta - Notre Dame (SCT28 \& SLKT), yellow - IOP)

\section{Observations and data reduction}

In this paper new and archival observations of J071126 are reported. New observations were obtained with the $1.2 \mathrm{~m}$ STELLA/WiFSIP telescope, from the Inastars Observatory Potsdam (IOP), with the $1.8 \mathrm{~m}$ Vatican Advanced Technology Telescope (VATT), with the newly inaugurated $80 \mathrm{~cm}$ Sarah L. Krizmanich telescope (SLKT) and with a $28 \mathrm{~cm}$ Schmidt-Cassegrain telescope (shorthand SCT28), both owned by the University of Notre Dame (Indiana). Archival observations were obtained with the VATT in 2009, by the Hankasalmi observatory (HO, Finland) and by Gerhard Dangl via his website 1 . The VATT data from 2009 were originally published by Thorne et al. (2010) but re-analyzed here in the same manner as all other data for the sake of homogeneity. The HO-data were retrieved from

\footnotetext{
1 http://www.dangl.at
} 


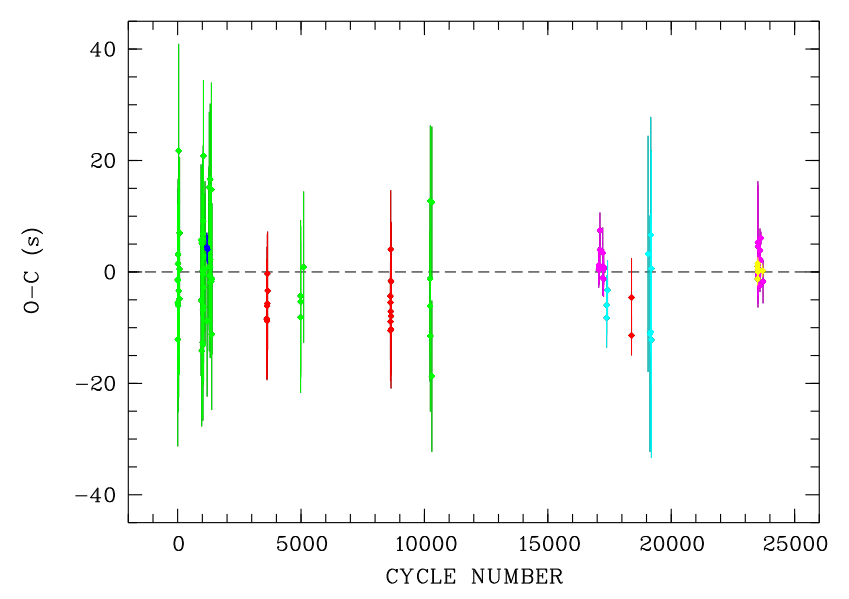

Fig. 2 Observed minus calculated times of eclipse centres of J071126. Colors are the same as in Fig. 1

the webpage of the observatory 2 . The whole data set comprises time-resolved photometric observations obtained during more than 50 nights at the various locations. Instead of listing every single night of observation that was used in this study we indicate each night for which we could derive an eclipse epoch in the CSS long-term light curve displayed in Fig. 1. Nevertheless, an overview of the facilities used and the main observational parameters is given in Table 1.

J071126 was put on the observing program of the Hankasalmi Observatory after its transient nature was reported and was intensively observed during 2009 and occasionally in 2010 and 2011. All those data were obtained with an integration time of $30 \mathrm{~s}$.

The observations by Dangl from Nonndorf (Austria) were recorded with a video system (for details see his webpage) and binned afterwards to a time resolution of $2 \mathrm{sec}-$ onds. A GPS-clock was directly connected to the videograbber. The timing accuracy per frame is $2.56 \mathrm{~s}$ (Dangl, private communication).

Details of the observations with the VATT in 2009 were described by Thorne et al. (2010) and the description will not be repeated here. Additional data in UBV bands were obtained during three nights in 2010 and in the R and Vilnius $\mathrm{S}$ bands in 2013.

Observations with an $28 \mathrm{~cm}$ Schmidt-Casegrain telescope (SCT28) owned by the University of Notre Dame were performed during eight nights in 2012 and four nights in 2014, mostly in white light but occasionally during two nights in 2012 through B and R filters, respectively.

The $80 \mathrm{~cm}$ SLKT, also owned by the University of Notre Dame, was used during two commissioning nights of the telescope for un-filtered observations with integration times as short as 2 seconds.

J071126 was observed from Inastars observatory Potsdam during four nights in March and April 2014. The instrument and the observational setup was the same as described in Schwope \& Thinius (2014).

2 http://murtoinen.dynds.org

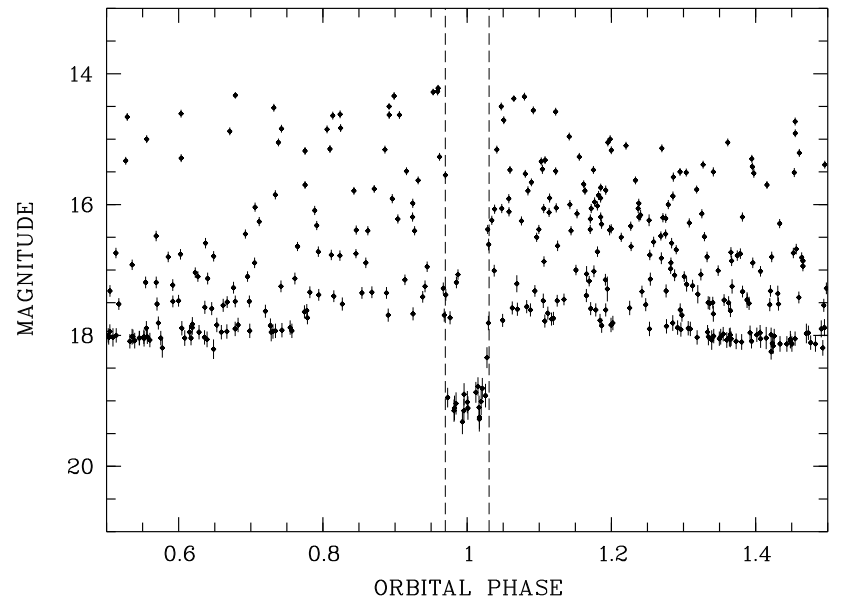

Fig. 3 CRTS data folded over binary phase

The robotic telescope STELLA-1 (aperture $1.2 \mathrm{~m}$, Strassmeier et al. 2004) is equipped with the wide-field imaging photometer WiFSIP and was used during two nights in November 2012 and during five nights in April 2013 for time-resolved photometric observations of J071126. All CCD-frames were taken through an SDSS gfilter.

The raw CCD-frames obtained from the various sites were bias-corrected, dark-subtracted and flat-fielded using calibration data obtained for this purpose. In the context of the current study the timing of individual CCD-frames is of utmost importance. The anticipated start of individual exposures is stored in the FITS-headers in the DATE-OBS keyword. However, CCD pre-flashes and other delays due to the operating system may give rise to a delayed shutter opening. Whenever possible, this shutter latency was determined and added to the measured times of eclipse ingress and egress. When this was not possible, a systematic error of any timing value was taken into account. Without measuring the shutter latency the widely used MaximDL CCD-software does not write decimal seconds to the FITS-headers but cuts time to the integer second. When integer seconds were written by the CCD software, half a second was added to the measured times and a systematic uncertainty of 0.5 seconds was added to the error budget. The measured or estimated shutter latencies together with the systematic timing uncertainties are listed in columns (7) and (8) of Tab. 1

Afterwards, differential photometry using ESO-MIDAS routines was performed with respect to comparison stars \#139 or \#142 on the AAVSO star chart (\#139: RA(J2000) $=7: 11: 13.17, \mathrm{DE}(\mathrm{J} 2000)=44: 03: 59.7, B=14.696, V=$ 13.918; \#142: $\operatorname{RA}(\mathrm{J} 2000)=7: 11: 35.50, \mathrm{DE}(\mathrm{J} 2000)=$ 44:04:03.3, $B=15.167, V=14.158)$. The default choice was to use star \#139. The flux ratios and differential magnitudes shown in Figs. 6 and 7 refer to that star.

The centroids of the stellar images of both the target and the comparison star were determined via two-dimensional Gaussian fits on a number of images with clean detections of both stars. The positions were used to determine the posi- 


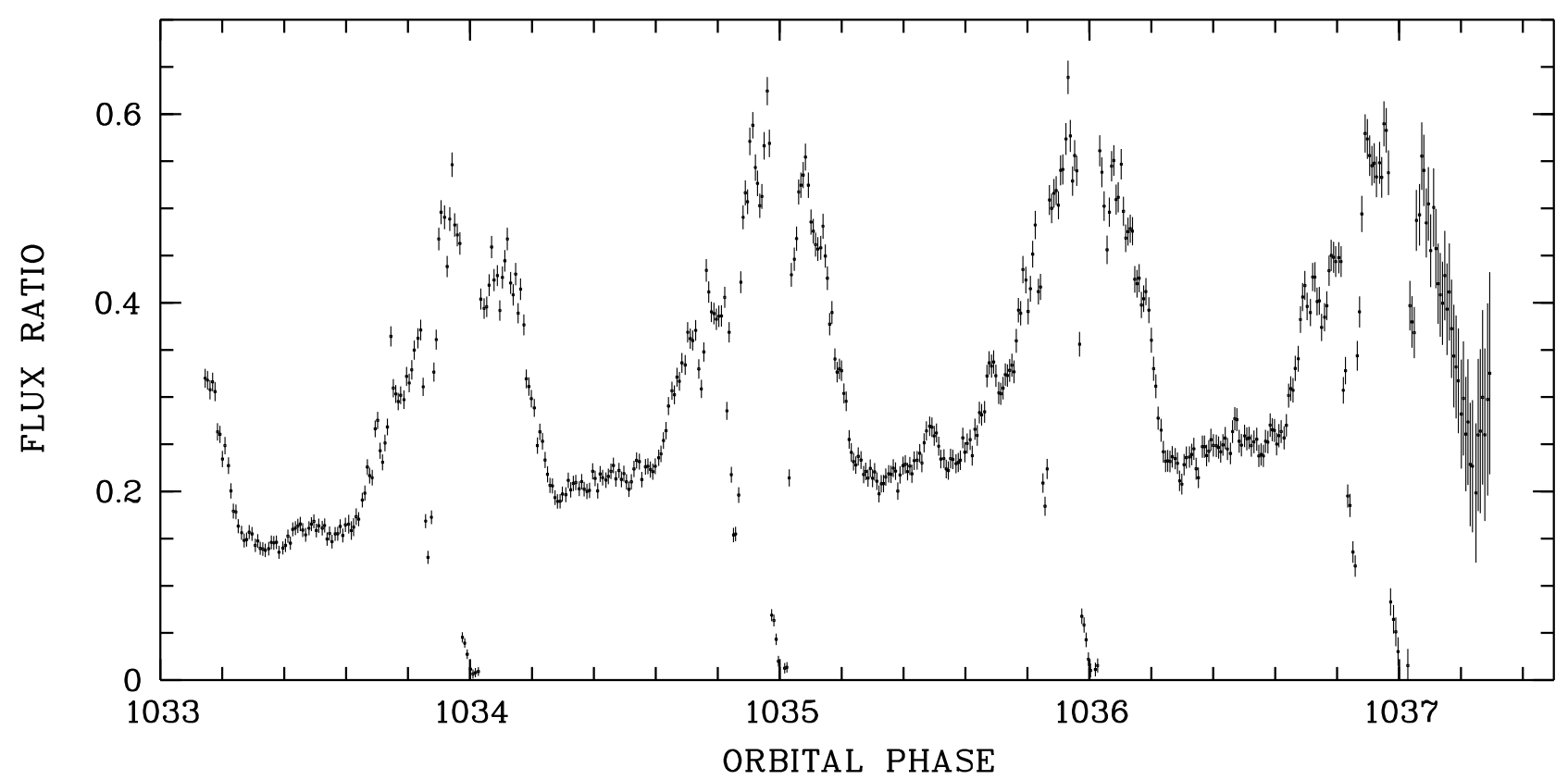

Fig. 4 Time-resolved photometry obtained on March 26, 2009, from Hankasalmi observatory. Shown is the flux ratio between the target and comparison \#139 from the AAVSO chart for the target.

tional offset in pixel units between the two stars. Then aperture photometry was performed on each CCD frame using concentric apertures for the object (plus sky background) and the sky background, centered initially on the comparison star, then at the offset position and always using the same apertures for target and comparison star. With this procedure a brightness measurement was possible even during eclipses of J071126, when the source became too faint for centering. Errors of individual measurements were determined taken into account the sky brightness, the object brightness and the read-noise properties of the CCD. The flux ratio between the target and the comparison star was further investigated for timing of the eclipse and for a discussion of the shape of the light curve(s).

\section{Analysis and results}

\subsection{A precise long-term ephemeris}

The times of all CCD frames were corrected to refer to midexposure, corrected for arrival at the solar system barycenter and converted to barycentric dynamical time (TDB) which equals terrestrial time (TT) at our precision.

The times of individual ingress and egress were measured by averaging a few data points before and after ingress/egress, computing the half-light intensity and reading the times with a cursor from a graph of the light curve (see Schwope \& Thinius 2014, for a graphical representation). Uncertainties of individual measurements were set to half the integration time of individual CCD frames, unless a data gap degrades the timing accuracy further. There were occasions when only one eclipse feature, either the ingress

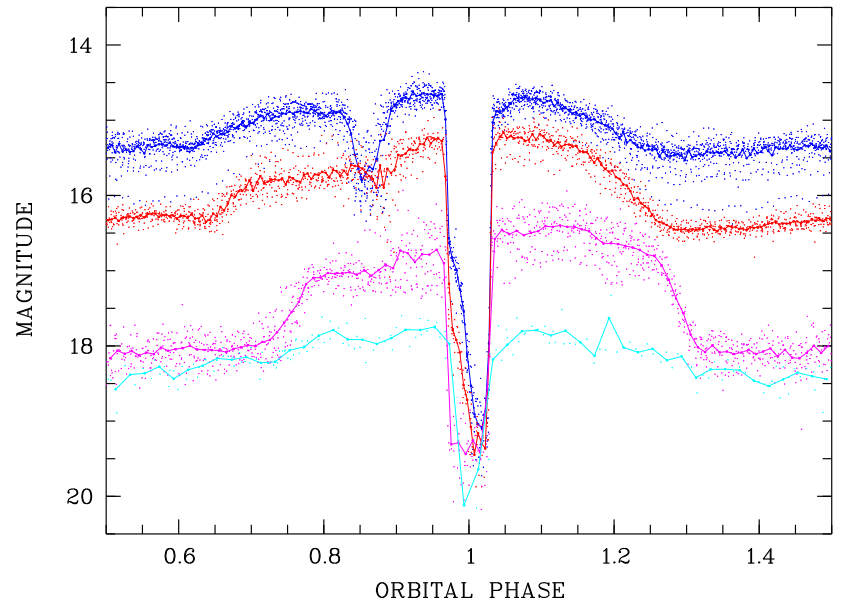

Fig.5 Phase-averaged light curves of J071126 obtained in 2009 from Hankasalmi observatory through high, intermediate, low and very low states, respectively. The data were averaged into 200,200,100, and 50 phase bins from top to bottom using the ephemeris of Eq. 2] (high state: March 26, 27, and April 1, 8, 14, 15, $22-25$; intermediate state: January 1, 2, 4, and March 19; low state: January 7, March 21, 24 , and 25; very low state: January 16, September 19, 20).

or the egress, could be determined. In order to include those eclipses in the analysis as well a two-stage process was chosen to derive timings of the eclipse centre. Firstly the mean eclipse length was determined using all fully covered eclipses. A weighted average of 102 individual measurements gave $\Delta t_{\mathrm{ecl}}=433.08 \pm 0.65 \mathrm{~s}$, which corresponds to $\Delta \phi=0.061596 \pm 0.000092$ phase units. Then the eclipse centers were determined from the timings of the ingress 


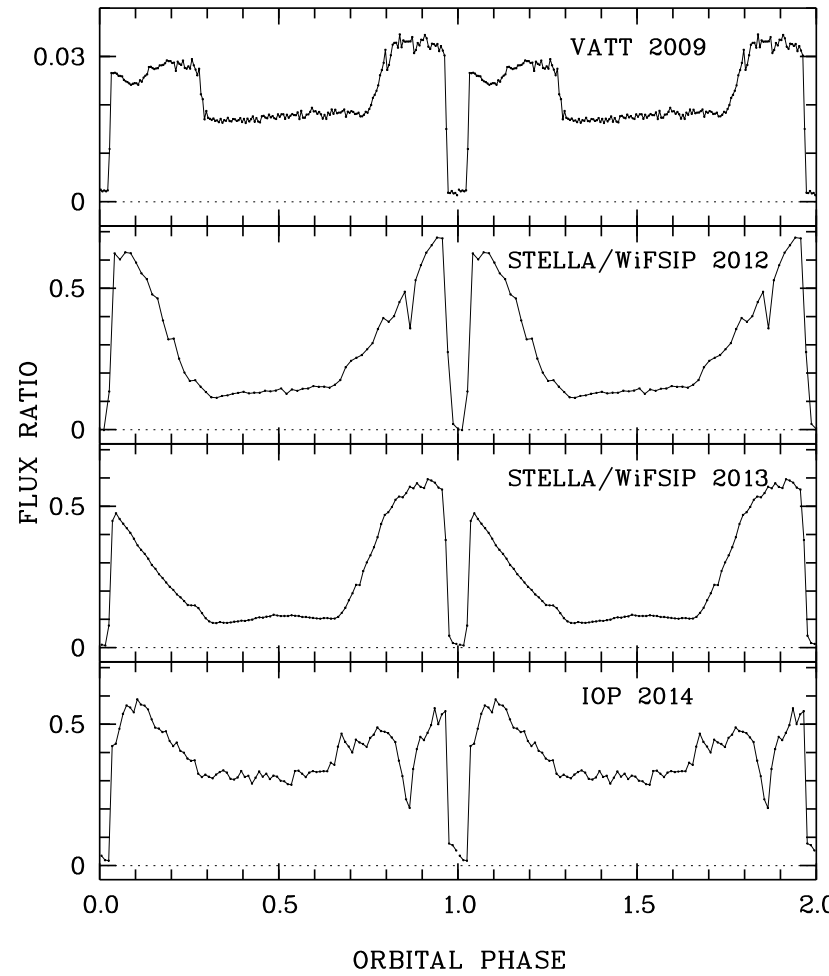

Fig. 6 Phase-averaged light curves of J071126 obtained between 2010 and 2014 from various sites. All data are shown twice for clarity and were phased using the ephemeris of Eq. 2 and averaged into 100 phase bins (66 phase bins for the 2012 observations with STELLA). Note the different scale used in the first panel.

and/or egress corrected for half the eclipse length. This procedure resulted in one (ingress or egress) or two (ingress and egress) independent measurements of the eclipse centre. In the latter case the finally accepted value was the weighted average of the two measurements. All eclipse timings are listed in Tab.2.

A weighted linear regression to all 109 data points yields the linear ephemeris for the eclipse centre

$$
\begin{array}{r}
\mathrm{BJD}(\mathrm{TDB})=2454833.207868(14)+ \\
E \times 0.0813768094(6)
\end{array}
$$

(numbers in parenthesis give formal $1 \sigma$ uncertainties, reduced $\chi_{\nu}^{2}=0.92$ for 107 d.o.f.). The residuals with respect to this linear fit are shown in Fig. 2, The diagram and the fitting statistics show that all data are in agreement with the linear fit.

\subsection{The photometric behavior of J071126 through high and low states}

\subsubsection{CRTS photometry}

The CSS long-term light curve shown in Fig. 1 covers the period between November 2005 and October 2013. The transient behavior was recognized in late 2008 but pronounced photometric variability is evident in archival data

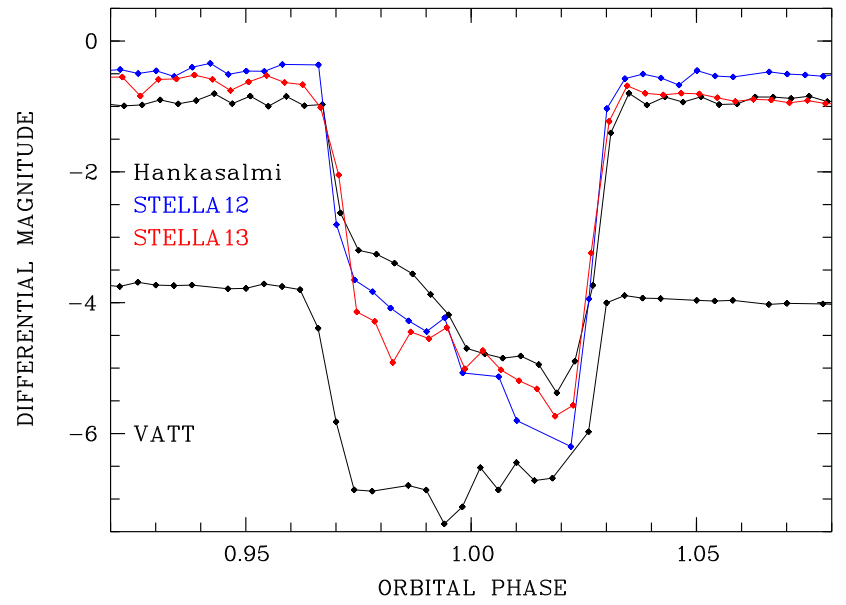

Fig. 7 Shape of the eclipse in high and low states.

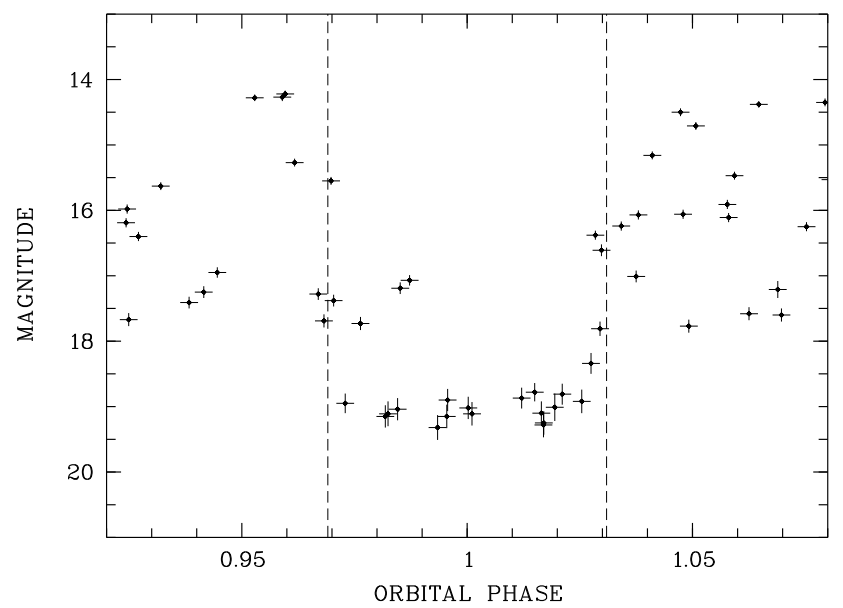

Fig. 8 CRTS data of CSS081231:071126+440405 folded over orbital phase.The diagram gives eclipse details, the length of the horizontal bars indicate the integration time per measurement. The dashed vertical lines indicate the measured eclipse length.

even prior to this. An initial low state at $V \sim 18$ was followed by a state of intermediate brightness between MJD 54000 and 54200 at $V \sim 16$ (September 2006 till April 2007). The object then returned to a low state before turning bright with $V_{\max }=14.2$ in late 2008. This was followed by another low state in the fall of 2009. J071126 was observed in intermediate to high states since then. Overall, the CSS-data obtained in white light show variability with an amplitude of about $5 \mathrm{mag}$, the maximum brightness is at 14.2, the minimum at 19.3. A considerable number of CSSmeasurements revealed the object at 19th mag, i.e. about one magnitude below the average low-state brightness.

Phase-folding of all CSS data (385 data points) gives further insight (see Figs. 3 and 8). It firstly shows that all the CSS measurements at 19th magnitude fall into the eclipse. The eclipse light curve is flat-bottom at $m_{\mathrm{CSS}} \simeq$ 19. This is about 1.9 mag brighter than the low-state Vband eclipse brightness measured by Thorne et al. (2010) at 


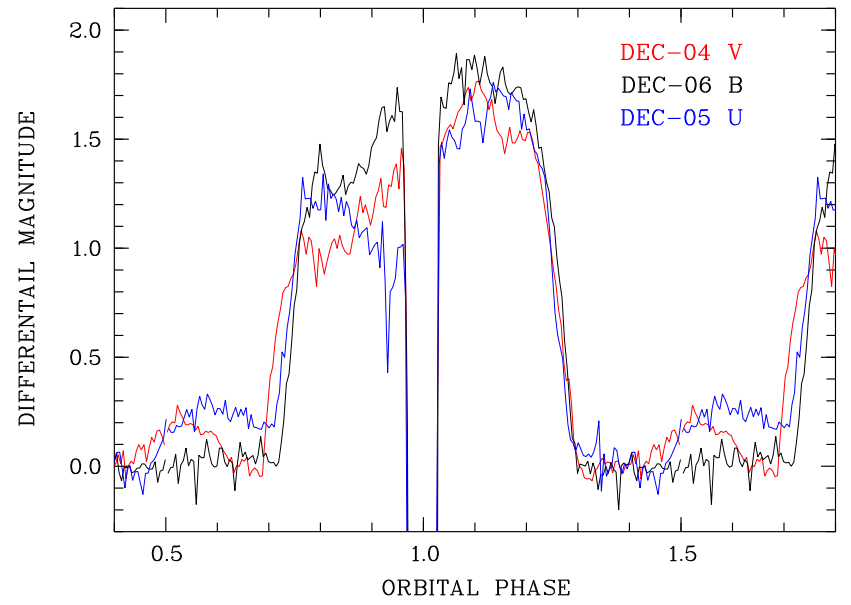

Fig. 9 UBV-photometry obtained with the VATT in October 2010. Shown are phase-averaged data (binsize 0.005 phase units, $\sim 35$ seconds). Differential magnitudes were corrected to the same mean value around phase 0.4 .

$V=20.86 \pm 0.05$. During the eclipse in the low state the remaining light originates mainly on the companion star. At a period of $117 \mathrm{~min}$ one expects $V-I=2.54$ if the donor follows the CV sequence described by (Knigge 2007). No color transformation into the CSS-system is available for such red stars, but given the red sensitivity of the CSSsystem it appears reasonable that both eclipse brightness measurements are compatible with each other.

In the CRTS the object displays out-of-eclipse variability between bright and faint phases with a minimum amplitude of about $0.2 \mathrm{mag}$.

\subsubsection{Multi-site phase-resolved photometry}

The orbital variability pattern in high and low states cannot be read from the CRTS data but only from phaseresolved observations. Time-resolved and phase-averaged light curves obtained by us are shown in Figs. 4, 5, and 6 Figures 4 and 5 contain only data from 2009 obtained at Hankasalmi. Phase-folded light curves from other observatories obtained between 2010 and 2014 are shown in Fig. 6. Plotted in Figures 4 and 6 is the flux ratio as a function of the orbital phase. The data shown in the top panel were obtained in a low state (Thorne et al. 2010). All other data in this Figure represent high accretion states.

The light curves show commonalities and some marked differences. All light curves, irrespective of the accretion state, display the alternating bright-/faint-phase pattern due to the self-eclipse of the accretion region (or regions).

The data obtained at Hankasalmi in the year 2009 can be sorted into four distinct accretion states. Transitions between different states are observed to occur on timescales of days or even a few binary cycles. The transition from an intermediate to a high state happened within two binary cycles, see Fig. 4
Changes of the overall brightness are accompanied by changes of the start, end, and length of the bright phase and the occurrence of a pre-eclipse dip. The eclipse light curves are also variable and will be discussed in the next section.

The length of the bright phase changes from $\Delta \phi=$ $\phi_{\text {end }}-\phi_{\text {start }}=1.30-0.75=0.55$ in the low state to $1.26-0.63=0.63$ phase units in the high state (see Figs. 5 and (6). The center of the bright phase thus moves from $1.03 \pm 0.02$ in the low state to $0.98 \pm 0.02$ in the intermediate and finally to $0.95 \pm 0.02$ in the high state, hence changing from a trailing spot at longitude $\psi \sim-10^{\circ}$ to a leading spot at $\psi \sim 18^{\circ}$. Some care should be taken when interpreting these numbers purely in geometrical terms, since the measured spot location may depend on the filter used and thus not necessarily indicate a change of the accretion geometry. This becomes clear from the VATT UBV photometry in December 2010 (Fig.9). Those data show a color-dependant start of the bright phase, whereas the end of the bright phase is not dependent on the filter. The accretion state is assumed to be the same.

Most of the high-state light curves show a more gradual increase of brightness at the beginning of the bright phase and a step decline into the self-eclipse of the accretion area. During the STELLA observation in 2013, however, the object displays the opposite behavior. At this epoch the otherwise prominent pre-eclipse dip has also vanished. Hence, in April 2013 the accretion geometry seemed to be rather different than at the other occasions in the high state.

The occurrence and the phase of the pre-eclipse dip is also dependent on the accretion state. The dip is typically present in the high state (but not during the STELLA observation in 2013) and has a typical depth of 1.4 mag. It is centered on phase 0.86 , but the phase interval $0.82-0.90$ is affected by absorption. In the intermediate state the dip is less pronounced and it seems to show a larger phase jitter. The average depth is 0.3 mag but may be as large as 1.5 mag. The phase interval $0.84-0.92$ is affected by absorption, the average dip occurs at a later phase than during the high state, $\phi_{c}=0.89$. No dip is detected during low and very low states.

Pre-eclipse dips are observed in several polars, a very prominent example being HU Aqr (Schwope et al. 2001). They are caused by absorption of radiation originating from the accretion column in the magnetically guided part of accretion stream (or accretion curtain). The orbital phase and the extent of this feature gives clues to the location of the coupling region and to the distribution of absorbing matter in the magnetosphere of the white dwarf. The absence of this feature at some occasions while previously present might thus indicate that the matter is accreted then by another region below the orbital plane.

The VATT V-band light curve obtained in 2009 (top panel in Fig. 6) is markedly different than the high state light curves. The rise to and the fall from the bright phase show a steep gradient. Also, there is a broad flux depression centered on phase 0.1 . Those properties of the light curve are 
reminiscent of WW Hor or DP Leo where the light curves were shaped by strong cyclotron beaming (Beuermann et al. 1990; Schwope \& Mengel 1997).

Occasionally a secondary maximum is observed during the faint phase (see e.g. the VATT V- and U-band light curves from 2010 in Fig. 9, the STELLA g-band light curve from 2013 in Fig. 6 and the white-light data obtained 2009, March 26, in Fig. 47. At other occasions the faint-phase brightness is more or less constant or just gradually increasing. This secondary hump has a maximum amplitude of 0.3 mag and is located at phase 0.50 (April 2013), 0.54 (October 2010, V), and 0.59 (October 2010, U), respectively. Various radiation components may contribute to the observed flux: a second accretion region, the accretion stream, the donor star, and the white dwarf. Photospheric emission from the stars appears unlikely to us because those radiation components should be more prominent during low accretion states.

\subsection{Eclipse light curves and binary parameters}

Figures 7 and 8 show eclipse details from phase-resolved observations presented here or from the CSS database. The same phase-resolved data were used as in Fig. 6 but with finer binning (0.004 phase units). During the low state the light curve in the eclipse is flat. It is thus reasonable to assume that the residual emission is due to the donor star (Thorne et al. 2010). During intermediate and high states rather intense emission is remaining throughout the eclipse of the white dwarf. This is due to the accretion stream/curtain outside the orbital plane. From its initial brightness after eclipse ingress it fades typically by two magnitudes but seems to stay above the brightness of the donor star, hence some parts of the stream are always visible.

Most of the CRTS data that were obtained the whitedwarf eclipse show the source at $V_{\mathrm{CSS}}=19$ but a few data points showed the object considerably brighter, $17.0-$ $17.7 \mathrm{mag}$. These data points were obtained during very high accretion states and the high brightness is also due to the bright accretion stream.

The eclipse width $\Delta \phi_{\text {ecl }}$, the orbital inclination $i$ and the mass ratio $Q$ are related to each other via the Roche geometry (Chanan et al. 1976). Taking the observed eclipse width at face value, i.e. as a proxy for the eclipse width of the white dwarf, a relation between between $i$ and $Q$ can be derived. If one further assumes that the donor star follows Knigge's sequence, $M_{2}=0.16 \mathrm{M}_{\odot}$ (Knigge 2007), and assuming $M_{1}=0.8 \mathrm{M}_{\odot}$, a typical value for the white dwarfs in CVs, the orbital inclination is $i=81.7^{\circ}$. For a standard single white dwarf, $M_{1}=0.6 \mathrm{M}_{\odot}$, the inclination would be $79.8^{\circ}$ and for a more massive white dwarf with $M_{1}=1.0 \mathrm{M}_{\odot}$ one gets $i=83.2^{\circ}$. A further uncertainty derives from the fact, that the observed eclipse width does not exactly correspond to the eclipse of the white dwarf centre of mass. It belongs mainly to the accretion spot somewhere on the surface of the white dwarf.

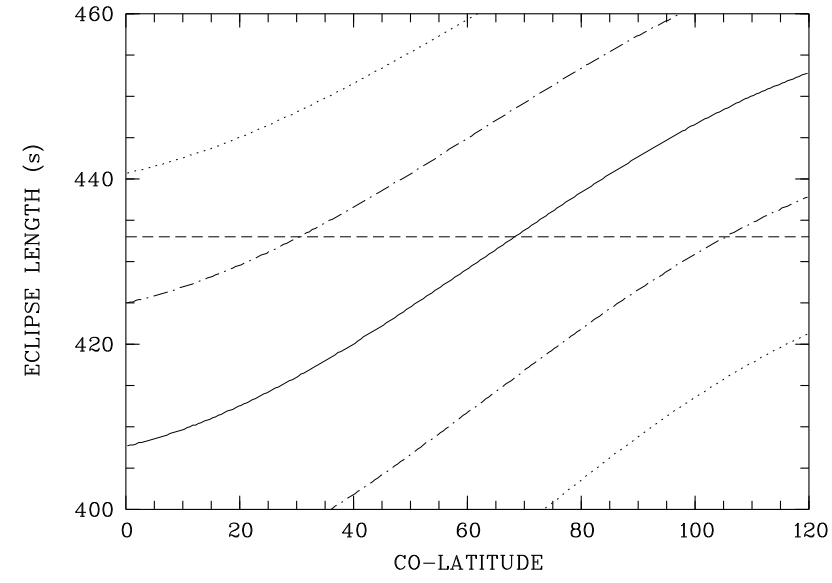

Fig. 10 Eclipse length of points along the central meridian for an $0.8 \mathrm{M}_{\odot}$ white dwarf as a function of the co-latitude $\beta$ and the orbital inclination. The measured eclipse length of $433.08 \mathrm{~s}$ is illustrated by the dashed horizontal line.

In order to estimate the size of this effect, we have calculated eclipse light curves for certain points on a sphere. A white dwarf with $0.8 \mathrm{M}_{\odot}$ was assumed and the points were chosen along the central meridian (meridian on the white dwarf through the rotation axis and the line connecting both stars). Eclipse light curves were computed as a function of the stellar co-latitude $\beta$, i.e. the angle between the rotation axis and the point on the meridian. Results are shown in Fig. 10 for $i=81.7^{\circ}, \pm 0.5^{\circ}, \pm 1.0^{\circ}$, respectively. The measured eclipse length is the same for an accretion spot at $\beta=68^{\circ}$ and at the center of mass of the assumed $0.8 \mathrm{M}_{\odot}$ white dwarf. The computations show that one should consider another $\sim 0.5^{\circ}$ systematic uncertainty of the inclination derived straight from the observed eclipse width via Roche-lobe geometry. The inclination is thus expected to be in the range $i=79.3^{\circ}-83.7^{\circ}$.

\section{Discussion and conclusion}

The present study presents an analysis of multi-epoch timeresolved photometric observations of the bright eclipsing polar CSS081231:071126+440405. We have established a precise long-term ephemeris based on 109 individual timings of the eclipse obtained between 2009 and 2014. We also determined a precise eclipse length of $433.08 \pm 0.65 \mathrm{~s}$.

An eclipse length of $408 \pm 7 \mathrm{~s}$ reported by Thorne et al. (2010) can definitely be ruled out already on the basis of a few light curves that were obtained with sufficient time resolution. The measured eclipse length was used to constrain the orbital inclination to lie within the range $i=$ $79.3^{\circ}-83.7^{\circ}$. The uncertainty is due to the unknown, hence assumed mass of the white dwarf and the unknown location of the accretion spot. Photometric observations with high time resolution during a low state would be most useful to directly measure the size of the white dwarf and high-speed photometric observations during a high state would allow 
to measure the size and location (azimuth) of the accretion spot directly.

We find a linear relation between cycle number and mideclipse time which is stable over a time interval of 5.3 years or almost 24000 orbital cycles of the binary. We estimate the maximum mass of an unseen companion by adding one higher polynomial order and explore the $\chi^{2}$ space until the fit deteriorates. We request a $95 \%$ confidence to reject the hypotheses of a quadratic term in the ephemeris. The bow of the parabola is used as a proxy for the amplitude of the sine curve due to the LTT effect. A period 5.3 years is assumed for the putative substellar companion. Its amplitude then is about $3 \mathrm{sec}$ ( $6 \mathrm{~s}$ peak-to-peak) which corresponds to $M_{\mathrm{pl}} \simeq 2 \mathrm{M}_{\mathrm{Jup}}$ for a negligible eccentricity, for an edgeon planetary system and for a total mass of the system of $1 \mathrm{M}_{\odot}$. Care has to be taken to not overinterpret this result. It may well be that the system is on an apparently linear part of the $O-C$ diagram over the past 5 years which over a longer time base might become saw-tooth shaped (for an example see NN Ser, Beuermann et al. 2010). Monitoring of the system for several years to come is needed to confirm or disprove the linear ephemeris. The current work defines the reference for such future studies.

Monitoring over long times is also needed to measure the center of the bright phase and probe the coupling mechanism and stability of the white dwarf orientation in the system. With a monitoring campaign lasting more than 30 years Beuermann et al. (2014) have uncovered the likely oscillation of the magnetic axis around an equilibrium position of the white dwarf in the eclipsing polar DP Leo. The spot longitude of DP Leo was observed at $-10^{\circ}$ to $-15^{\circ}$ some 30 years ago, similar to what is observed in J071126. Contrary to DP Leo the longitude of the accretion spot in J071126 is observed to vary as a function of the mass accretion rate. In the low state the spot lies at $-10^{\circ}$, and in the high state at $+10^{\circ}$. The accretion-rate dependent location of the accretion spot complicates the proposed investigation. The high brightness of J071126 on the other hand is promising to establish a large database and to separate oscillations of the magnetic axis and spot migrations.

The final part of the paper is devoted to a discussion of the possible accretion geometry. An accretion arc is thought to arise at the foot points of dipolar field lines connecting the ballistic accretion stream with the white dwarf. Possible solutions are sought with the following constraints: - the accretion spot is centered on phase 0.95 in the high state and 1.03 in the low state. Thus the spot longitude is $\psi=+18^{\circ}$ in the high state and $\psi=-10^{\circ}$ in the low state (measured with respect to the line connecting both stars in a coordinate system centered on the white dwarf); - the inclination is between $i=79.3^{\circ}$ and $83.7^{\circ}$; - in the high state parts of the accretion stream are never completely obscured by the donor star; - the stagnation region is extending from about $35^{\circ}$ to about $65^{\circ}$ in azimuth in the high state, and from $\sim 30^{\circ}$ to $\sim 60^{\circ}$ in the intermediate state (phase interval of pre-eclipse dip). The absence of pre-eclipse dips in the low state prevents us from constraining the stagnation region when the accretion rate is further reduced.

Firstly we are discussing a possible high-state accretion geometry. The presence of a pre-eclipse dip requires an accretion region above the orbital plane. An only partial occultation of the accretion stream requires a sufficiently low orbital inclination and a sufficiently high elevation of the stream. The latter becomes possible if the inclination of the magnetic axis is sufficiently small. A location of the accretion region closer to the line connecting both stars than the stagnation region means that the magnetically guided part of the stream has to be strongly re-directed in the frame of the binary.

We use our own visualization software to test different accretion scenarios. The original free-falling stream is eventually guided by magnetic field lines towards an accretion region on the white dwarf. The coupling region is found by balancing the ram and the magnetic pressure along the stream. In the coupling region the stream is assumed be instantaneously re-directed and traveling along dipolar field lines. We find a possible accretion geometry for the high state assuming a co-latitude and an azimuth of the magnetic axis of $\beta=18^{\circ}$, and $\psi=-3^{\circ}$, and an inclination of $79.3^{\circ}$. For the chosen orientation of the magnetic axis the accretion stream would be completely occulted at phase zero if the orbital inclination were higher. A higher inclination would be possible if the magnetic axis were more closely aligned to the rotation axis. In such a geometry the magnetically guided part cannot be re-directed to feed an accretion region close to the line connecting both stars. A sketch of the accretion geometry is given in Fig. 11 where the binary is seen at orbital phases 0.9 , i.e.at the end of the pre-eclipse dip, and at phase 0.0 .

At a lower accretion rate coupling occurs earlier and the magnetically guided part of the stream is lifted further above the plane. Hence, at lower accretion rate the magnetically guided part is not expected to be completely covered by the donor during eclipse phase. The pre-eclipse dip occurs later in phase, i.e. closer to the eclipse, and consequently the accretion region lies closer to the meridian. All those expectations are met in the intermediate state. Hence, the high and intermediate states can be explained with the same accretion geometry.

The same arguments apply if the accretion rate is further reduced, i.e. if one wants to understand the geometry in the low state. One might expect to always see some remaining radiation from the magnetic stream lurking at high elevations above the orbital plane. Contrary to this a flat eclipse light curve is observed suggesting that matter in the magnetosphere is completely covered by the donor star during eclipse in the low state. Also, the observed location of the accretion spot, $\psi_{\text {spot }} \simeq-10^{\circ}$ is incompatible with the chosen orientation of the magnetic axis, $\psi=-3^{\circ}$. If a more extreme value of $\psi$ is chosen, $\psi<10^{\circ}$, the high-state geometry cannot be explained. 

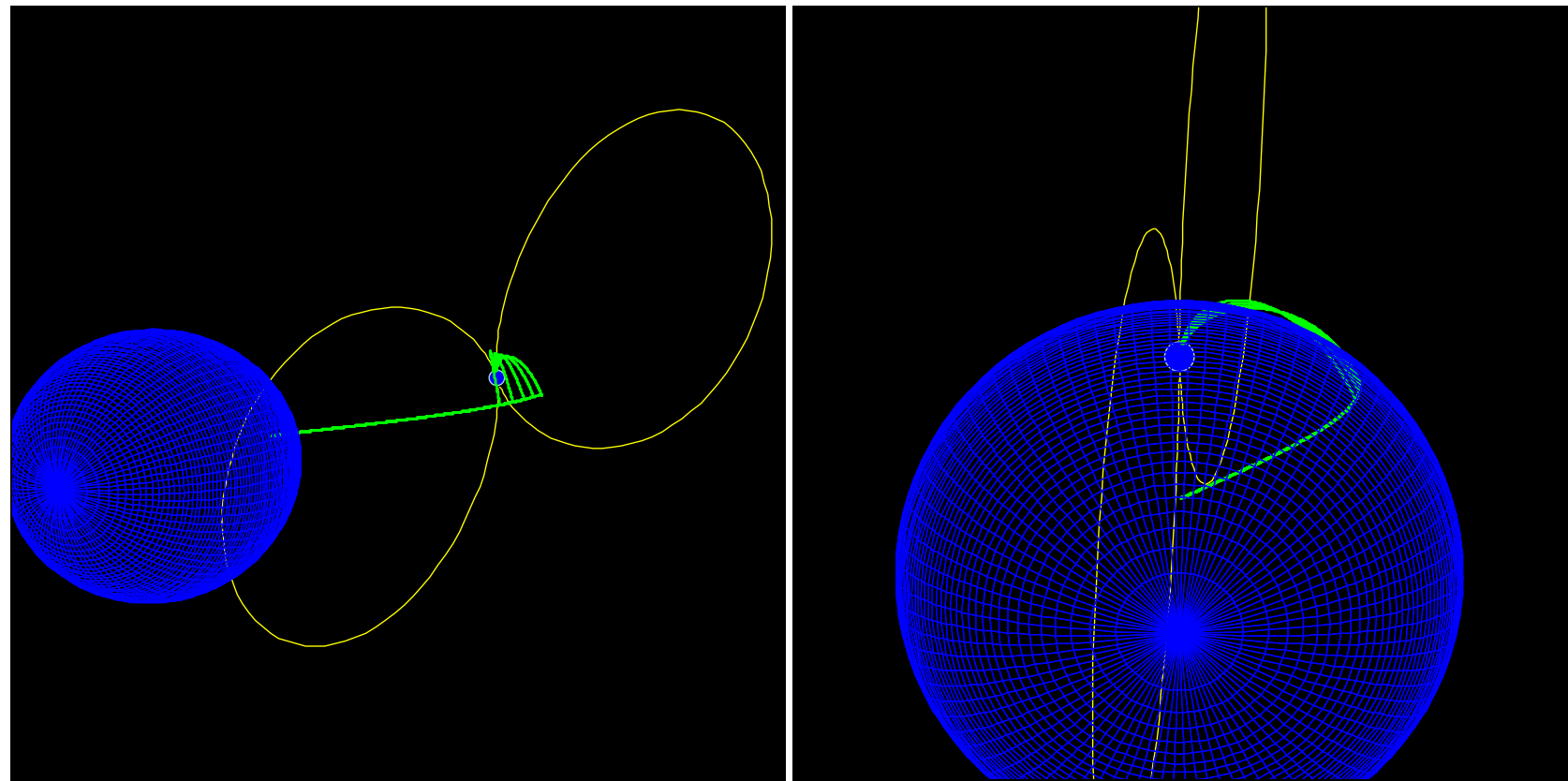

Fig. 11 Possible accretion geometry of CSS081231:071126+440405 in a high state seen at binary phases 0.9 and 1.0 . The green lines illustrate accreting field lines, the yellow lines are dipolar field lines through the magnetic meridian thus illustrating the orientation of the magnetic field. The orbital inclination is $79.3^{\circ}$, the mass ratio $Q=5$ and the mass of the white dwarf $M_{\mathrm{WD}}=0.8 \mathrm{M}_{\odot}$.

In short, we do not find a solution which satisfies all observational constraints during high and low accretion states. A possible remedy might be found in assuming a nondipolar field structure. A further variation of the theme would be assuming that accretion takes place at a region below the orbital plane in the low state. This would still require a non-dipolar field structure but would explain the flat eclipse light curves. The absence of the pre-eclipse dip in the STELLA light curves obtained in 2013 remains puzzling.

Acknowledgements. We thank an anonymous referee for helpful comments.

We thank Gerhard Dangl for obtaining and providing his photometric data.

This study is based partly on data obtained with the STELLA robotic telescope in Tenerife, an AIP facility jointly operated by AIP and IAC.

We thank the Krizmanich family for their generous donation of the Sarah L. Krizmanich telescope to the University of Notre Dame.

Based on observations with the VATT: the Alice P. Lennon Telescope and the Thomas J. Bannan Astrophysics Facility. We thank Richard Boyle and the Vatican Observatory for scheduling the VATT observations.

The CSS survey is funded by the National Aeronautics and Space Administration under Grant No. NNG05GF22G issued through the Science Mission Directorate Near-Earth Objects Observations Program. The CRTS survey is supported by the U.S. National Science Foundation under grants AST-0909182 and AST1313422 .

\section{References}

Beuermann, K., Dreizler, S., Hessman, F. V., \& Schwope, A. D. 2014, A\&A, 562, A63

Beuermann, K., Hessman, F. V., Dreizler, S., et al. 2010, A\&A, 521, L60

Beuermann, K., Thomas, H.-C., Schwope, A. D., Giommi, P., \& Tagliaferri, G. 1990, A\&A, 238, 187

Bours, M. C. P., Marsh, T. R., Breedt, E., et al. 2014, MNRAS , 445, 1924

Chanan, G. A., Middleditch, J., \& Nelson, J. E. 1976, ApJ , 208, 512

Drake, A. J., Djorgovski, S. G., Mahabal, A., et al. 2009, ApJ , 696, 870

Goździewski, K., Nasiroglu, I., Słowikowska, A., et al. 2012, MNRAS , 425, 930

Horner, J., Marshall, J. P., Wittenmyer, R. A., \& Tinney, C. G. 2011, MNRAS , 416, L11

Katysheva, N. \& Shugarov, S. 2012, Mem. Soc. Astron. Italiana, 83,670

Knigge, C. 2007, MNRAS , 382, 1982

Qian, S.-B., Liu, L., Liao, W.-P., et al. 2011, MNRAS , 414, L16

Schwarz, R., Schwope, A. D., Vogel, J., et al. 2009, A\&A, 496, 833

Schwope, A. D. \& Mengel, S. 1997, Astronomische Nachrichten, 318,25

Schwope, A. D., Schwarz, R., Sirk, M., \& Howell, S. B. 2001, A\&A, 375, 419

Schwope, A. D. \& Thinius, B. D. 2014, Astronomische Nachrichten, 335, 357

Strassmeier, K. G., Granzer, T., Weber, M., et al. 2004, Astronomische Nachrichten, 325, 527

Thorne, K., Garnavich, P., \& Mohrig, K. 2010, Information Bul- 
letin on Variable Stars, 5923, 1

Wittenmyer, R. A., Horner, J., Marshall, J. P., Butters, O. W., \& Tinney, C. G. 2012, MNRAS , 419, 3258 
Table 2: Cycle number and mid-eclipse times of J071126

\begin{tabular}{|c|c|c|c|}
\hline Cycle & $T_{C}$ in $\mathrm{BJD}$ (TDB) & $\Delta T_{C}$ & Observatory $^{a}$ \\
\hline 0 & 2454833.20785 & $1.6 \mathrm{E}-4$ & 1 \\
\hline 1 & 2454833.28923 & $1.6 \mathrm{E}-4$ & 1 \\
\hline 2 & 2454833.37055 & $1.6 \mathrm{E}-4$ & 1 \\
\hline 3 & 2454833.45186 & $2.2 \mathrm{E}-4$ & 1 \\
\hline 16 & 2454834.50993 & $1.6 \mathrm{E}-4$ & 1 \\
\hline 17 & 2454834.59129 & $1.6 \mathrm{E}-4$ & 1 \\
\hline 18 & 2454834.67258 & $2.2 \mathrm{E}-4$ & 1 \\
\hline 40 & 2454836.46319 & $2.2 \mathrm{E}-4$ & 1 \\
\hline 41 & 2454836.54428 & $2.2 \mathrm{E}-4$ & 1 \\
\hline 74 & 2454839.22969 & $1.6 \mathrm{E}-4$ & 1 \\
\hline 75 & 2454839.31113 & $1.6 \mathrm{E}-4$ & 1 \\
\hline 76 & 2454839.39258 & $1.6 \mathrm{E}-4$ & 1 \\
\hline 948 & 2454910.35315 & $1.6 \mathrm{E}-4$ & 1 \\
\hline 949 & 2454910.43440 & $1.6 \mathrm{E}-4$ & 1 \\
\hline 973 & 2454912.38734 & $1.6 \mathrm{E}-4$ & 1 \\
\hline 974 & 2454912.46894 & $1.6 \mathrm{E}-4$ & 1 \\
\hline 1009 & 2454915.31692 & $1.6 \mathrm{E}-4$ & 1 \\
\hline 1010 & 2454915.39844 & $1.6 \mathrm{E}-4$ & 1 \\
\hline 1022 & 2454916.37481 & $1.6 \mathrm{E}-4$ & 1 \\
\hline 1023 & 2454916.45626 & $1.6 \mathrm{E}-4$ & 1 \\
\hline 1024 & 2454916.53780 & $1.6 \mathrm{E}-4$ & 1 \\
\hline 1034 & 2454917.35159 & $1.6 \mathrm{E}-4$ & 1 \\
\hline 1035 & 2454917.43294 & $1.6 \mathrm{E}-4$ & 1 \\
\hline 1036 & 2454917.51423 & $1.6 \mathrm{E}-4$ & 1 \\
\hline 1037 & 2454917.59568 & $1.6 \mathrm{E}-4$ & 1 \\
\hline 1046 & 2454918.32825 & $1.6 \mathrm{E}-4$ & 1 \\
\hline 1048 & 2454918.49069 & $1.6 \mathrm{E}-4$ & 1 \\
\hline 1049 & 2454918.57211 & $1.6 \mathrm{E}-4$ & 1 \\
\hline 1107 & 2454923.29200 & $1.6 \mathrm{E}-4$ & 1 \\
\hline 1108 & 2454923.37340 & $1.6 \mathrm{E}-4$ & 1 \\
\hline 1182 & 2454929.39531 & $3.0 \mathrm{E}-5$ & 2 \\
\hline 1194 & 2454930.37167 & $1.6 \mathrm{E}-4$ & 1 \\
\hline 1206 & 2454931.34834 & $3.0 \mathrm{E}-5$ & 2 \\
\hline 1267 & 2454936.31234 & $1.6 \mathrm{E}-4$ & 1 \\
\hline 1280 & 2454937.37036 & $1.6 \mathrm{E}-4$ & 1 \\
\hline 1281 & 2454937.45159 & $1.6 \mathrm{E}-4$ & 1 \\
\hline 1282 & 2454937.53293 & $1.6 \mathrm{E}-4$ & 1 \\
\hline 1316 & 2454940.29976 & $1.6 \mathrm{E}-4$ & 1 \\
\hline 1317 & 2454940.38110 & $1.6 \mathrm{E}-4$ & 1 \\
\hline 1318 & 2454940.46269 & $1.6 \mathrm{E}-4$ & 1 \\
\hline 1319 & 2454940.54390 & $1.6 \mathrm{E}-4$ & 1 \\
\hline 1366 & 2454944.36857 & $1.6 \mathrm{E}-4$ & 1 \\
\hline 1367 & 2454944.45013 & $2.2 \mathrm{E}-4$ & 1 \\
\hline 1380 & 2454945.50773 & $1.6 \mathrm{E}-4$ & 1 \\
\hline 1391 & 2454946.40299 & $1.6 \mathrm{E}-4$ & 1 \\
\hline 3609 & 2455126.89667 & $1.2 \mathrm{E}-4$ & 3 \\
\hline 3610 & 2455126.97805 & $1.2 \mathrm{E}-4$ & 3 \\
\hline 3621 & 2455127.87319 & $1.2 \mathrm{E}-4$ & 3 \\
\hline 3622 & 2455127.95460 & $1.2 \mathrm{E}-4$ & 3 \\
\hline 3634 & 2455128.93112 & $8.2 \mathrm{E}-5$ & 3 \\
\hline 3635 & 2455129.01256 & $8.2 \mathrm{E}-5$ & 3 \\
\hline 3646 & 2455129.90767 & $1.2 \mathrm{E}-4$ & 3 \\
\hline 4980 & 2455238.46428 & $1.6 \mathrm{E}-4$ & 1 \\
\hline 4981 & 2455238.54570 & $1.6 \mathrm{E}-4$ & 1 \\
\hline 4982 & 2455238.62707 & $1.6 \mathrm{E}-4$ & 1 \\
\hline 5102 & 2455248.39235 & $1.6 \mathrm{E}-4$ & 1 \\
\hline 8621 & 2455534.75729 & 4.1E-5 & 3 \\
\hline 8622 & 2455534.83859 & $6.2 \mathrm{E}-5$ & 3 \\
\hline
\end{tabular}


Table 2: Cycle numbers and mid-eclipse times of J071126 (continued)

\begin{tabular}{|c|c|c|c|}
\hline Cycle & $T_{C}$ in BJD(TDB) & $\Delta T_{C}$ & Observatory $^{a)}$ \\
\hline 8623 & 2455534.92003 & $6.2 \mathrm{E}-5$ & 3 \\
\hline 8624 & 2455535.00136 & $1.2 \mathrm{E}-4$ & 3 \\
\hline 8634 & 2455535.81515 & $1.2 \mathrm{E}-4$ & 3 \\
\hline 8635 & 2455535.89666 & $1.2 \mathrm{E}-4$ & 3 \\
\hline 8636 & 2455535.97797 & $1.2 \mathrm{E}-4$ & 3 \\
\hline 8646 & 2455536.79164 & $1.2 \mathrm{E}-4$ & 3 \\
\hline 8647 & 2455536.87304 & $1.2 \mathrm{E}-4$ & 3 \\
\hline 8648 & 2455536.95449 & $1.2 \mathrm{E}-4$ & 3 \\
\hline 10226 & 2455665.36704 & $1.6 \mathrm{E}-4$ & 1 \\
\hline 10227 & 2455665.44848 & $1.6 \mathrm{E}-4$ & 1 \\
\hline 10239 & 2455666.42488 & $1.6 \mathrm{E}-4$ & 1 \\
\hline 10240 & 2455666.50654 & $1.6 \mathrm{E}-4$ & 1 \\
\hline 10300 & 2455671.38878 & $1.6 \mathrm{E}-4$ & 1 \\
\hline 10301 & 2455671.47052 & $1.6 \mathrm{E}-4$ & 1 \\
\hline 17065 & 2456221.90311 & $3.7 \mathrm{E}-5$ & 5 \\
\hline 17075 & 2456222.71689 & $3.7 \mathrm{E}-5$ & 5 \\
\hline 17076 & 2456222.79827 & $3.7 \mathrm{E}-5$ & 5 \\
\hline 17112 & 2456225.72787 & $3.7 \mathrm{E}-5$ & 5 \\
\hline 17113 & 2456225.80928 & $3.7 \mathrm{E}-5$ & 5 \\
\hline 17224 & 2456234.84201 & $3.7 \mathrm{E}-5$ & 5 \\
\hline 17225 & 2456234.92344 & $5.3 \mathrm{E}-5$ & 5 \\
\hline 17259 & 2456237.69022 & $3.7 \mathrm{E}-5$ & 5 \\
\hline 17260 & 2456237.77157 & $3.7 \mathrm{E}-5$ & 5 \\
\hline 17261 & 2456237.85298 & $3.7 \mathrm{E}-5$ & 5 \\
\hline 17381 & 2456247.61809 & $6.2 \mathrm{E}-5$ & 4 \\
\hline 17382 & 2456247.69949 & $6.2 \mathrm{E}-5$ & 4 \\
\hline 17418 & 2456250.62909 & $6.1 \mathrm{E}-5$ & 4 \\
\hline 18390 & 2456329.72733 & $8.2 \mathrm{E}-5$ & 3 \\
\hline 18391 & 2456329.80863 & $4.1 \mathrm{E}-5$ & 3 \\
\hline 19062 & 2456384.41264 & $2.5 \mathrm{E}-4$ & 4 \\
\hline 19136 & 2456390.43435 & $2.5 \mathrm{E}-4$ & 4 \\
\hline 19172 & 2456393.36392 & $2.5 \mathrm{E}-4$ & 4 \\
\hline 19173 & 2456393.44550 & $2.5 \mathrm{E}-4$ & 4 \\
\hline 19197 & 2456395.39847 & $2.5 \mathrm{E}-4$ & 4 \\
\hline 19198 & 2456395.47970 & $2.5 \mathrm{E}-4$ & 4 \\
\hline 23499 & 2456745.48149 & $1.3 \mathrm{E}-5$ & 6 \\
\hline 23500 & 2456745.56289 & $1.3 \mathrm{E}-5$ & 6 \\
\hline 23510 & 2456746.37666 & $1.3 \mathrm{E}-5$ & 6 \\
\hline 23511 & 2456746.45803 & $1.3 \mathrm{E}-5$ & 6 \\
\hline 23513 & 2456746.62084 & $1.2 \mathrm{E}-4$ & 5 \\
\hline 23514 & 2456746.70220 & $1.3 \mathrm{E}-4$ & 5 \\
\hline 23522 & 2456747.35317 & $1.3 \mathrm{E}-5$ & 6 \\
\hline 23525 & 2456747.59729 & $4.5 \mathrm{E}-5$ & 5 \\
\hline 23526 & 2456747.67867 & $4.5 \mathrm{E}-5$ & 5 \\
\hline 23599 & 2456753.61920 & $1.9 \mathrm{E}-5$ & 7 \\
\hline 23599 & 2456753.61921 & $4.5 \mathrm{E}-5$ & 5 \\
\hline 23600 & 2456753.70053 & $1.3 \mathrm{E}-5$ & 7 \\
\hline 23600 & 2456753.70060 & $4.5 \mathrm{E}-5$ & 5 \\
\hline 23636 & 2456756.63019 & $1.3 \mathrm{E}-5$ & 7 \\
\hline 23707 & 2456762.40788 & $1.3 \mathrm{E}-5$ & 6 \\
\hline 23722 & 2456763.62851 & $4.5 \mathrm{E}-5$ & 5 \\
\hline
\end{tabular}

a) Observatory key: 1 - Hankasalmi, 2 - Nonndorf, 3 - VATT, 4 - STELLA , 5 - SCT28, 6 - IOP, 7 - SLKT 